Research article

\title{
Biological complexity, quantum coherent states and the problem of efficient transmission of information inside a cell
}

\author{
Alexey V. Melkikh* \\ Ural Federal University, Mira Street 19, Yekaterinburg 620002, Russia
}

\section{A R T I C L E I N F O}

\section{Article history:}

Received 18 December 2012

Received in revised form 31 January 2013

Accepted 6 February 2013

\section{Keywords:}

Information channel

Protein conformations

Quantum coherent states

Intracellular calculation

\begin{abstract}
A B S T R A C T
The intracellular channel of information transmission was analyzed from the point of view of complexity. The most important steps in the transfer of information within a cell are the folding, transport and recognition of proteins. It was shown that the large number of conformational degrees of freedom that proteins possess can paradoxically lead to an information channel with an exponentially small capacity. To resolve this paradox, a model, which assumes a quantum collective behavior of biologically important molecules, was proposed. Experiments to test the quantum nature of the intracellular transfer of information were also proposed.
\end{abstract}

(c) 2013 Elsevier Ireland Ltd. All rights reserved.

\section{Introduction}

The transmission, processing and storage of information in a living cell have a number of significant features. Among those features, the following can be noted: the complexity of the information molecules (e.g., proteins and RNA) and the complexity of the transport systems that transport these molecules. The problem with these systems is the following: the carriers of information that should send a signal at a fixed place often have a large number of degrees of freedom, which implies that the probability of information transmission losses can be high.

Information loss can occur both at the stage of protein folding and at the stages of protein transport and recognition. These losses can result from faulty conformations or reactions with other macromolecules.

In the papers (Melkikh and Seleznev, 2012; Melkikh and Sutormina, 2013) was formulated the problem of transport of macromolecules and molecular interactions. It is shown that existence of the unique way of proteins folding, and for the interaction between macromolecules leads to a contradiction.

The aim of this paper is to propose a quantum mechanism for the interaction of biologically important molecules. This mechanism avoids the contradiction that arises between an exponentially large number of possible protein conformations and the need to select a small number of these conformations to

\footnotetext{
* Tel.: +73433759349.

E-mail addresses: melkikh2008@rambler.ru, mav@dpt.ustu.ru
}

maintain a high capacity of intracellular and intercellular information channels.

\section{Complexity of macromolecules, problem of protein folding and intracellular transmission of information}

Consider an information transmission channel within the cell, including the synthesis, folding, transport and delivery (e.g., the regulation of gene activity) of a protein to a specific location. Each stage of an information channel includes a large number of variants, of which, as a rule, only one corresponds to the transfer of the correct signal. Consider the most important events that regulate this process.

\subsection{Protein folding}

Proteins are first synthesized as a linear molecule, but most proteins may properly function only in a single (native) configuration. The problem of protein folding to achieve the native configuration is called Levinthal's paradox. Despite the large number of works on Levinthal's paradox (e.g., Zwanzig et al., 1992; Berezovsky and Trifonov, 2002; Finkelstein and Ptitsyn, 2002; Bai, 2003, 2006; Grosberg and Khokhlov, 2010), the final solution of the paradox has not been clarified. The basic assumption in the solution of the paradox lies in the fact that each protein should be folded through a specific mechanism that provides a rapid folding process. However, the existence of such a mechanism is not proven; this existence is also not based on first-principles calculations for specific proteins or on the general laws of statistical physics. The problem of the 



Fig. 1. Equivalent states in one-component system at third step of folding.

origin of such a path is not discussed. According to a number of authors (e.g., Berezovsky and Trifonov, 2002), the typical number of steps required for the folding of a protein that consists of 150 domains, each of which can have three possible states, is approximately

$3^{150} \approx 10^{72}$

According to Berezovsky and Trifonov (2002), the enumeration of these options would require approximately $10^{48}$ years, which is unacceptable for the cell and does not correspond to the experimental data.

However, if the first path to the native configuration requires astronomical time (when this path did not exist), how did a specific mechanism of folding arise for each protein?

Why is the existence of a special folding mechanism for each protein not obvious? On one hand, each protein has a specific function (e.g., a part in the catalysis processes and ion transport); thus each protein should have a certain amino acid composition. On the other hand, the amino acid composition should adhere to strict requirements to ensure that the protein folds rapidly (this problem will be discussed below). These two tasks (the execution of the function and rapid protein folding) are quite different, and the requirements for the composition of the protein are also different. It follows that the same amino acid sequence should meet different (and likely opposite) requirements.

Consider the folding of a chain of monomers (the chain represents the protein). Consider first the case in which all of the monomers are identical and that the chain is folded in twodimensional space.

For example, the first four monomers can have the following configuration after folding (the other monomers in the chain have not yet undergone folding and are not shown):

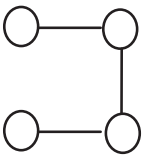

This configuration occurs only if both the monomers are attracted to each other and the connections between the monomers can rotate. If the connection between the monomers is rigid or if repulsion dominates, the folding will not be beneficial and thus may not be realized. These cases will not be considered further.

There are two equivalent capabilities for the next step in the folding process (i.e., there is a divergence (or fork) in the folding path), as shown in Fig. 1:

Fig. 1 shows that two states are equivalent in terms of the interaction energy and the distances between the nearest monomers.
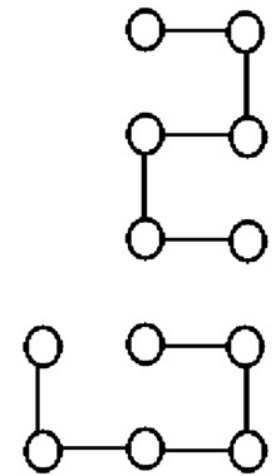

Fig. 2. Equivalent states in one-component system at fourth step of folding.

Consequently, according to statistical physics, the two states should be equally likely. In this analysis, we assumed that each monomer interacts only with its immediate neighbors. This assumption is valid because the known experimental data on the interaction potential between atoms is on the order of the inter-atomic distance in a solid and the size of the monomers considered in this analysis (amino acid residues) is larger than the size of atoms.

The next folding step leads to several variants, two of which are equivalent in energy (and hence in probability), as shown in Fig. 2:

After these two configurations, the folding path diverges due to the equivalent energies of the next two conformations. For example, the states shown in Fig. 3 should be equivalent in energy.

Thus, we can conclude that divergences exist in most steps of the folding process of a single-component system of monomers. In this case, the probabilities of the existence of each of the two states (and thus the two divergent paths) are the same. If we consider that the native configuration is unique, the chances that the chain will be folded correctly at the $n$th step of the folding process is.

$P(n)=\left(\frac{1}{2}\right)^{n / 2}$

For sufficiently large $n$, this value will be exponentially small.

Now consider a two-component system that is composed of two different monomers. There are three different interaction potentials between the monomers (which are of type 1 and 2): $U_{11}, U_{12}$ and $U_{22}$.

The two-component system can be ordered (the different monomer types are alternated in the chain) or unordered. It is easy to show that divergences occur frequently in the folding

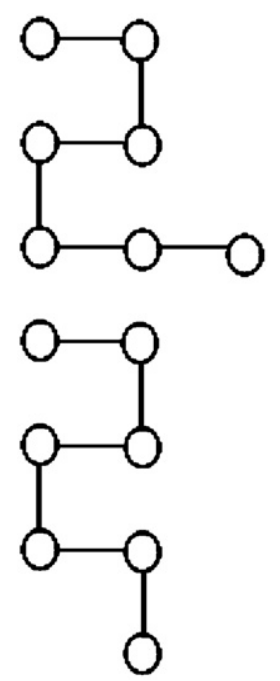

Fig. 3. Equivalent states in one-component system at fifth step of folding. 


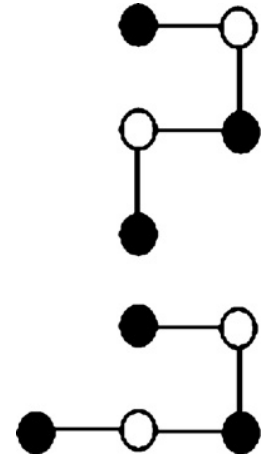

Fig. 4. Two equivalent states in two-component system.

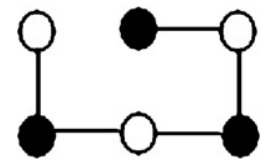

Fig. 5. Control of the folding by different potentials.

mechanism of a two-component system. In fact, if the initial configuration has the form

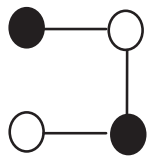

there are two equivalent states in the next folding step (there is a divergence in the folding process), as shown in Fig. 4:

Note that if the monomers of one type repel each other, the chain will not fold.

The next step in the folding process involves interactions between two different monomers with different energies. If these energies are similar to each other, the realization of this interaction will be likely, which indicates that the system will behave almost as a single-component system with an exponentially large number of possible configurations.

The control of the folding process is required if the interaction energies of between monomers varies. For example, if $U_{11} \ll U_{12}$, then the probability that the state shown in Fig. 5 is realized in the next step of the folding process is overwhelming.

Nevertheless, there is another divergence in the folding process in the following step. Thus, the number of possible configurations of a chain remains exponentially large, and the probability of any given configuration (native) being realized is exponentially small.

Consider now an aperiodical two-component system. We shall vary the structure of a chain to obtain different spatial configurations. It is easy to use examples to show that it is impossible to avoid the appearances of divergences in the folding process of this system because the probability that two identical monomers will be present in this type of chain is high. Consider the following initial configuration:

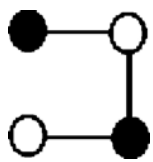

The following folding step would give rise to two states with equivalent energy, as shown in Fig. 6.

If the two-component system has sufficiently long sections composed of one type of monomer, the folding process will approximately the folding of a one-component system.

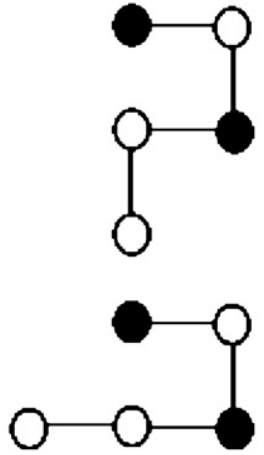

Fig. 6. Two equivalent states in two-component aperiodical system.

Consider the most general system: a long chain composed of $n$ different types of monomers with different interaction energies. At first sight, the large number of different components could allow the generation of sufficiently complex selection rules that could ensure that the folding process provides a unique variant.

However, the fact that the chain consists of many different types of monomers gives rise to an additional contradiction. The presence of different monomers implies the presence of different energy levels (at the limited value of a full energy interval). Thus, the energy spectrum becomes almost continuous, which decreases the control of the selectivity of the folding process. This loss of control will arise because the probability that the energy of alternative conformations will slightly differ will be high, i.e., the probability that particles with strongly differing potentials are found close to each other will be small.

If that the process demands that the interaction energies differ, the multi-component system can be reduced to a two-component system because the monomers can be divided into two types based on their energy.

For example, if general difference between the interaction energies is approximately $5-10 \mathrm{kT}$ (this value is typical for interactions between amino acids during protein folding), the energy difference between alternative conformations between 20 different monomers will be small. This result means that the probabilities that alternative conformations exist are comparable, which will result in a large number of possible configurations of the macromolecule.A similar problem exists in the growth of quasicrystals, which are a type of aperiodic structure (e.g., Bindi et al., 2009; Steinhardt, 2008; Macia, 2006). One of the possible growth mechanisms of quasicrystals is Penrose mosaics. However, it is unclear how these mosaics can result from the interaction of atoms. The literature does not describe any calculations on the growth of quasicrystals that are based on the real potentials of the interactions between atoms. Thus, the growth of quasicrystals involves an exponentially large number of possible atom configurations that do not correspond to the correct quasicrystal. It remains unclear the rules that forbid the formation of these chaotic configurations that do not result in the formation of the quasicrystal. If such an interdiction is not present, the probability of the growth of the correct quasicrystal appears to be exponentially small.

It is easy to show that the analysis of protein folding in three-dimensional space will result in an even greater number of divergences in the folding mechanism because additional equivalent energy states will appear due to the opportunity of folding along an additional axis.

Also note that other variants in the process can be considered because the macromolecule can start to fold from an arbitrary point (or from several points simultaneously) instead of from the end of the chain. It is natural that this will result in an even greater number of possible configurations. 
In addition, even if we assume that the rapid folding of an arbitrarily long chain that is composed of several types of monomers is possible, the following question is still unsolved: what such chain is capable of performing useful work or of transmitting helpful information? To be able to perform useful work (and/or transmit helpful information), the protein molecule should have a certain structure and configuration (native), which means that the replacement of one amino acid or a change in the order of the amino acids in the macromolecule will result in the protein being unable to perform its function effectively. However, it does not follow that this type of useful structure will result in the rapid folding of the macromolecule because the requirements for effective work and rapid folding are different.

Thus, the analysis of the folding mechanisms of macromolecules gives the following conclusions:

- The folding of a long chain of any structure inevitably produces a large number of divergences, which are accompanied by an exponentially large number of possible configurations. Thus, the probability of achieving the unique native configuration of a macromolecule is exponentially small.

- Even if there is a configuration that can be obtained within a relatively short folding time, it does not follow that this configuration can perform useful work.

Taken together, these conclusions imply that the losses in the transmission of information within a cell are large because of the folding of structures that are not able to transmit a specified signal.

\subsection{Transport of large molecules}

After the protein is folded, it must be transported through the active transport systems to a specific location in the cell. However, the transport of molecules involves a large number of degrees of freedom, as was considered in a previous paper (Melkikh and Seleznev, 2012). Most proteins are delivered to their destination through systems of active transport. In particular, the transport of proteins and RNA through the nuclear pores plays an important role in the life of a cell. For efficient operation, the transport vehicles must be designed such that all other possible reactions that are not related to the transport of specific proteins to specified locations are prohibited.

However, it is not clear how the transport of proteins is realized because the amino acids that make up proteins are dipoles or charged (either negatively or positively). What law can prevent the interaction of charged particles? If there is no such prohibition, the transport system will undergo many "parasitic" reactions or perhaps not work at all. It should be noted that this is still an open question.

According to Melkikh and Seleznev (2012), a problem arises in the transport of proteins and mRNAs from the nucleus. The transport system needs to be able to identify all of the proteins that are synthesized by the cell and transport these to specific locations in the cell.

The recognition of proteins is important not only for the transport of proteins to their destination but also for the sorting of the proteins that are transported from the endoplasmic reticulum to the Golgi complex or autophagosomes, which are cell compartments that remove abnormal protein complexes. The fact that these complexes exist suggests that different proteins interact with each other. However, how does the cell control this huge number of errors if the newly formed complex will result in new reactions that will, in turn, result in other new reactions, etc.? This complex forms a network of proteins that are unable to achieve their native configurations and are found in configurations with a long-lived local energy minimum.
How do autophagosomes distinguish abnormal protein complexes and incorrectly folded proteins? To be able to achieve this distinction, autophagosomes should have receptors that target specific proteins. In principle, these receptors could exist for all proteins, although there are thousands of different proteins in a cell. However, the number of molecules of each receptor would be small, if not only one. The information processing speed in such a system is then unclear because the machine that corrects errors, also might have errors, and these errors cannot be completely excluded.

However, the main problem is the mechanism by which different conformations of the same protein are recognized. As mentioned above, these possible conformations (of the total number of proteins) are exponentially many; thus, the surfaces that are available for the corresponding receptors are simply not sufficient. It is also necessary to have a mechanism that recognizes the correctly folded proteins. Then, how does a receptor have to be arranged to ensure that it recognizes only one type of protein, destroys any "bad" proteins, and does not react with the "right" proteins of other species (of which there are thousands)? Thus, the receptor will have very strict requirements in the sense of complexity, i.e., it will have a very large number of controlled degrees of freedom. Thus, the mechanism of this receptor is far from being understood.

From the point of view of the efficiency of transport processes (Melkikh and Seleznev, 2012), we can say that the problem in the transport of macromolecules is the elimination of ineffective ("parasitic") reaction channels. In the presence of an exponentially large number of such channels, the efficient transport of these molecules will tend to zero.

If at least one of these problems remains unresolved, it could result in an error catastrophe in the cell: one error in the transport of molecules will result in other errors, which will result in additional errors, and so on. At present, there are no models that attempt to answer these questions in the literature. Thus, errors in the transport of large molecules will reduce the capacity of intracellular information channels by orders of magnitude.

\subsection{Recognition of molecules}

The last important step is the localization of the protein at the destination. This step should include the recognition of a molecule-transporter before the transmission of information can be considered complete. The problem of recognition is relevant for the transport of molecules, although it must be analyzed in a broader context (see Section 2.2). Recognition is important not only for the transport of molecules but also for most of the biochemical reactions that occur in a cell. Many proteins act as the catalysts of biochemical reactions. In fact, the speed of some reactions can be accelerated by up to $10^{19}$-fold (e.g., Garcia-Viloca et al., 2004), and the correct recognition of molecules plays a key role in this acceleration.

For example, the receptors that are associated with G-proteins form a large family of transmembrane receptors. These receptors function as activators of intracellular signaling pathways, which eventually results in a cellular response. Receptors of this family are found only in eukaryotic cells: yeast, plants, choanoflagellates and animals. The ligands that bind and activate these receptors include hormones, neurotransmitters, light-sensitive substances, odorous substances, and pheromones; thus, these ligands vary in size from small molecules and peptides to proteins (King et al., 2003; Filmore, 2004).

It is believed that many chemical reactions (including those involved in the transmission of information) utilize the "lock and key" principle (or "hand-glove", e.g., Savir and Tlusty, 2007). This principle assumes that the shape of one molecule corresponds exactly to the shape of the other molecule. Thus, the (enzymatic) 
reaction only takes place if there is complete coincidence between the reacting molecules. If there is no match, the reaction likely (with overwhelming probability) does not occur. However, how does each "key" find its "lock"? There must be a large number of possible "keys" and "locks" at any specific time within a cell, and the probability that these respond to each other in any way, is, although not zero, less than that observed in the case of complete coincidence. This issue was considered in a previous paper (Melkikh and Sutormina, 2013).

Melkikh and Sutormina (2013) showed that the existence of a special interaction mechanism between individual protein domains and between large molecules is contradictory. Consider two areas of interaction between atoms (ions) belonging to different molecules: zone I (with a 3-5 $)$, in which

$U_{12} \geq k T$

and zone II, in which

$U_{12} \leq k T$

If two large molecules are in zone II, their interaction is too small to induce their movement. In this zone, the random interaction with water molecules has a central role, and the movement of large molecules is chaotic (non-directional). Thus that the reaction between these macromolecules will not meet the "key-and-lock" principle, and the molecules can form intermolecular complexes with other properties.

If two large molecules approach each other such, that the characteristic energy of interaction between their atoms becomes comparable to kT (zone I), the interaction between the molecules is significant. In this case, the selectivity of this interaction can be large, which means that the coincidence of the "key" and "lock" potential energy well that characterizes this interaction will be deep.

The fact that there are exponentially many of these potential energy wells is an important nuance. However, most of these potential energy wells will be only slightly less deep than the "basic" well. If an atom (ion) finds a local energy minimum, the atom (ion) cannot "feel" exactly where the global minimum is. In this situation, the depth of the global minimum is not important. In the theory of stochastic processes, this problem is called "task of the first achieving" (e.g., Van Kampen, 2007).

Melkikh and Sutormina (2013) noted that the number of atoms in zone I (first coordination sphere) may be very small (approximately six). However, the number of variants of such atoms is too small compared with the total number of large molecule conformations, which means that a molecule can only see a few surface atoms, and not the composition, of the other molecule during their collision, thereby preventing recognition.

The cell has several thousand molecules of different proteins, each of which attempt to react, in general, with all other proteins, which will lead to a large number of errors in the interaction of large molecules.

Cherstvy (2011) considered the interactions of proteins and DNA, the mechanisms of which are largely not understood. The model of protein recognition is based on the spatial coincidence of oppositely charged groups of DNAs and proteins. The depth of the "recognition well" was 3-10 kT. The question of what occurs during partial matches is not considered; however, based on the proposed model, we can conclude that the mismatch of one charge will result in a well depth decrease of approximately $10 \%$. Because we are considering approximately 10 charged groups, the different variants will include a significant number of mismatches. Accordingly, there will be a large number of potential wells, the depth of which will be only slightly less than the depth of the main well.

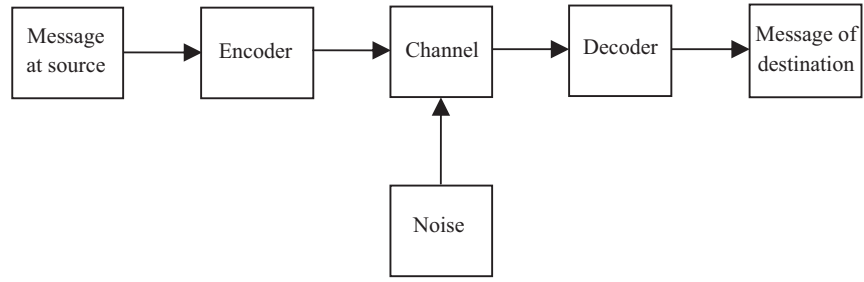

Fig. 7. Shannon's system of information transmission.

The interaction of proteins with each other has been repeatedly observed (e.g., the physical aspects of this interaction are discussed by Meszaros et al. (2011)). The different physical mechanisms of protein interactions have been discussed. It has been noted that the interaction of proteins often changes their conformations. The Gibbs energy plays a major role in the thermodynamics of protein interactions. From a thermodynamics point of view, the rate of reaction between proteins is described by the standard formula:

$K=\exp \left(-\frac{\Delta G}{k T}\right)$

However, note that Eq. (1) is valid only when the system has visited all of its states, i.e., at large times. If, however, the interaction of proteins has a characteristic time on the order of seconds, the time required to explore all the conformations of this complex is exponentially large, which means that formula (1) cannot be applied. As mentioned above, the problem then takes a different meaning: it will be the "task of the first achievement", in which the depth of the potential well is not important.

Meszaros et al. (2011) examined the same classical model of a "lock and key" interaction and the energy landscape of proteins. In particular, these researchers considered a funnel-like landscape that allows the protein to quickly reach its final configuration.

However, it remains unclear how such a landscape arises and in which cases it can be implemented.

To summarize the possible mechanisms by which information can be lost in the transport of macromolecules, we can offer an analogy with the Shannon single-channel communication system, which is shown in Fig. 7.

Note that protein folding, protein transport (either active or diffusion-controlled) and "key-and-lock" chemical reactions are largely analogous to coding, the transfer of information over the line of communication and decoding. It is important to note information can be lost in all of these stages.

The importance of Shannon's approach to the transfer of information in the cell has been previously observed (e.g., Waltermann and Klipp, 2011; Gorlich et al., 2011), but the physical cause of the loss of information, as well as the magnitude of these losses, have not been discussed.

Thus, based on the analysis of the transfer of information through macromolecules (proteins) within a cell, it can be concluded that such a channel must have an exponentially small capacity. To resolve this paradox, consider the possible quantum effects in the transmission of information within a cell.

\subsection{Entangled quantum states of biologically important molecules as a possible mechanism for the solution of the paradoxes}

Let us discuss the possibility that the paradoxes discussed in part 1 may have a solution in the framework of quantum mechanics. Many authors have repeatedly highlighted the importance of quantum effects in biological processes. Although these effects are associated with the possibility of the parallel processing of quantum information, some aspects of the movement and 
transformation of energy in biomolecules have a quantum nature. Let us consider some of these effects in more detail.

Quantum entanglement plays an important role in biological processes. For example, Davies (2004) examined the role of quantum mechanics in the processes of life. In particular, Davies noted that the uncertainty principle limits the precision of the quantum clock (Wigner inequality). The same logic can be applied to the process of protein folding. Basis on this analogy, the limit of the folding time was obtained:

$T<\frac{m_{0} a^{2} N^{3}}{\hbar}$

where $m_{0}$ is the mass, $a$ is the characteristic size of an amino acid and $N$ is the number of amino acids. Thus, the folding time will be proportional to $N^{3}$. According to Davies, the typical size of protein $l$ is equal to $a N$. This analysis is accurate only for relatively short proteins. For longer proteins, the characteristic size will be the diameter of the protein, and the characteristic time is thus proportional to $N^{7 / 3}$. The comparison of the characteristic time of protein folding that was calculated based on quantum constraints and the times that were measured experimentally shows good agreement between the times.

Davies argues that quantum mechanics plays a nontrivial role in various life processes, e.g., the physics of the polymerase is not fully understood. In particular, it is unclear what defines the normal speed of this machine. If we assume that the rate of work is determined by the Wigner inequality, it turns out that the experimental data do confirm this assumption. Davies suggested experiments to test the hypothesis of a quantum mechanism. He also considered a number of other molecular machines (e.g., actomyosin and proton pump) and suggested that their work is also determined by the Wigner inequality.

In this regard, we can observe that many other intracellular molecular machines perform similar work and are therefore subject to the same restrictions. It is possible that, for example, the rate of transport of large molecules is determined by the same inequality.

Patel (2001) suggested that the genetic code is a consequence of the quantum search algorithm. Based on Grover's algorithm, Patel concluded that the effectiveness of the quantum search (equal to $\sqrt{N}=2$ ) will be two-fold higher than that of the classical search. However, this entanglement of nucleotides must persist a sufficiently long time.

Davies et al. (2012) proposed a quantum model of intracellular metabolism. It is suggested that metabolic instability can lead to aging and cancer. According to Davies et al. (2012), metabolic energy can be stored in the coherent state of the oscillators in mitochondria. In this case, the state of the oscillator can be described by quantum theory, similarly to the Debye quantum theory of solids.

Goswami and Todd (1997) and Ogryzko (1997) have proposed that adaptive mutations may be generated by the environmentinduced collapse of the quantum wave function that describes DNA as a superposition of mutational states. For such a mechanism to be feasible, the evolving DNA wave function must remain coherent sufficiently long for it to interact with the cell's environment.

McFadden and Al-Khalili (1999) examined the quantum model of adaptive mutations, which occur in cells that are rarely divides. These mutations are time-dependent, of the number of divisions and appear after the cells are exposed to the environment.

MacFadden and Al-Khalili examined a population of cells with genes $A$ and $B$. If the proton of one of the bases is in a superposition of its states, the superposition persists after DNA replication. The cell will eventually be a superposition of the mutated and unmutated states. The authors considered a model of decoherence and show that decoherence in E. coli can exist for a long time (tens of seconds).
The authors considered the quantum Zeno effect, which shows that the measurement freezes the system in the initial state. There is also the reverse quantum Zeno effect, in which certain measurements can induce movement.

This last remark is fundamental because, as will be shown below, the relationship of quantum information and movement can play an important role in the maintenance of coherent states.

Matsuno and Paton (2000) reviewed the various aspects of quantum information in a living cell, such as recording, storing and processing. In particular, based on estimates of the characteristic times that the actin-myosin complex is found in muscles, Matsuno and Paton concluded that this complex has a very low effective temperature (on the order of $10^{-3} \mathrm{~K}$ ). This finding indicates that the wavelength that can be associated with the particle is large $(4.5 \mathrm{~nm})$. Thus, Matsuno and Paton concluded that quantum mechanics plays an important role in this molecular machine.

Hameroff (2003) suggested that a network of microtubules plays the role of quantum computer within the cell (neuron). In this case, the tubulin monomers act as elementary quantum cells, which are called qubits. The author also considered the problem of decoherence, which was one of the principal problems addressed in the manuscript. In particular, he considered a global topology and quantum error correction as opportunities to maintain a coherent state. However, the modeling of the microtubule network is similar to that explored in Section 1: how is such a complex system controlled? Why does a microtubule network not entangle (in terms of "parasitic" reactions)? The answer to these questions may also be related to the quantum properties of particles (monomers) and their long-range correlations.

The quantum aspects of brain activity were also discussed in a previous article (Beck and Eccles, 1992). Beck suggested that the release of neurotransmitters from synaptic vesicles is determined by quantum effects. In this case, the switching mechanism that he considered was the motion of quantum quasiparticles. It is assumed that quantum long-range interactions exist between synaptic vesicles. Note that the transport of synaptic vesicles themselves also represents a problem. How does the directed movement of synaptic vesicles controlled within a cell? One can assume that this directed motion also involves quantum mechanisms.

Let us also note a number of articles that address the problem of measurements in biological systems and the related problem of decoherence (Igamberdiev, 1999, 2004).

As a result of the analysis of quantum effects in the transfer of information and the transformation of energy in cells, it can be concluded that the main limiting factor is decoherence. If the time of decoherence is small, the quantum effects will play a minor role. In fact, decoherence prevents the creation of quantum computers.

However, we can pay attention to one substantial increase in the time of decoherence. Imagine a situation in which a particle (molecule) of mass $m$ encounters forces that induce it to move directionally with speed v. If this speed is low, the uncertainty principle argues that the position and the wave behavior of the particle are largely uncertain. In this case, the De Broglie wave length can be large. However, quantum uncertainty in the position of a particle in space can lead to a significant change in its interaction with the environment. If these two processes reinforce each other, as illustrated in Fig. 8, the decoherence time can be large.

Thus, the basic assumption with which the solution of the proposed paradoxes can be found is that some of the coherent quantum states of the intracellular molecules are abnormally stable. It can be assumed that the structure of biological molecules involves additional selection rules for quantum particles, which significantly slow decoherence. It is possible that coherent quantum states and entanglement of quantum states arise even in the synthesis of proteins (and DNA and RNA) and are then stored and maintained at the expense of non-local interactions. We can consider the 


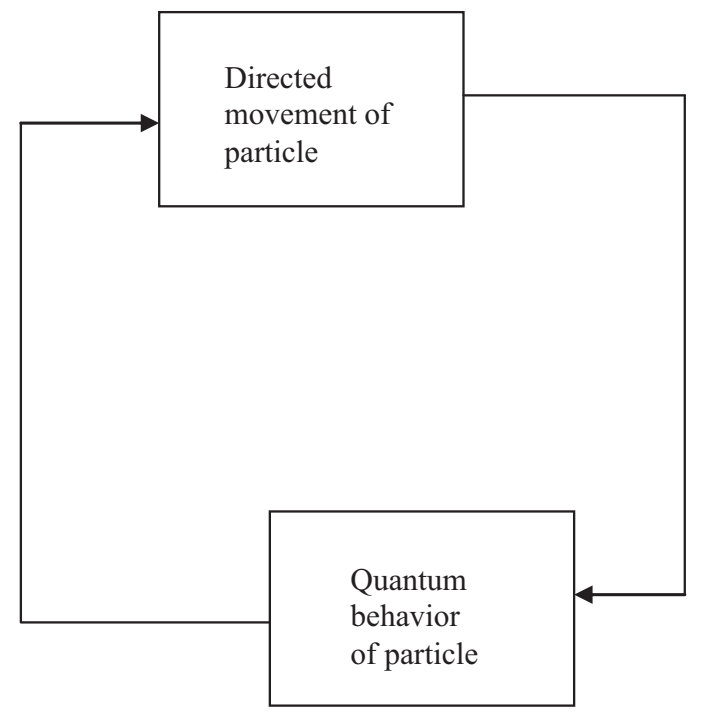

Fig. 8. Feedback and maintenance of coherent states.

entanglement of quantum states as a feedback mechanism that allows the biologically important molecules to move directionally. Although, entanglement (as a measure of order), is generally a resource for thermodynamic work, it may be, in particular, the work of the directed motion of particles along certain paths.

Summarizing the assumptions that various authors have made on the behavior of the quantum properties of proteins, we can assume that most proteins behave as quantum particles in a coherent state. An output of this state of superposition could be the result of some event (measurement), which could be related to the formation of the "right" structure. There may be a collapse of the wave function as a result of symmetry breaking.

Based on the above, we consider a model of energy conversion in intracellular processes.

1. The basic biomolecules (proteins, DNA and RNA) inside a cell are in coherent states. As a result, some of the processes (e.g., folding, motion and recognition) can be performed in parallel along many paths.

2. The continued existence of the coherent state is due to the fields of interaction between the molecule and to the fact that certain collective quantum states of molecules are very stable.

3. As a result, a set of proteins in a cell forms a quantum condensate, the existence of which is possible as a result of the positive feedback and symmetry breakage of the incoherent state.

4. This quantum state allows a parallel search of a large number of folding, recognition and transport variants. After the "right" configuration is found, the wave function collapses. As a result, the paradoxes discussed in Section 1 can be resolved.

Let us discuss the implications of these provisions and the possibility of experimentally verifying the hypothesis.

\section{Discussion: possible experiments for the verification of the hypothesis}

There is some analogy between the proposed model of quantum processes in the cell and the other quantum phenomena. A simple analogy is an atom with stable electron orbitals and quantum numbers; however, an analogy with the most intimate and well-studied quantum phenomena (superfluidity and superconductivity) can also be made. Table 1 shows the similarities and differences between the proposed quantum behavior of
Table 1

Similarities and differences between behavior of biologically important molecules and the phenomena of superfluidity and superconductivity.

\begin{tabular}{|c|c|}
\hline Cell processes & Superfluidity and superconductivity \\
\hline $\begin{array}{l}\text { A large number of particles are } \\
\text { in the coherent state }\end{array}$ & $\begin{array}{l}\text { A large number of particles are in the } \\
\text { coherent state }\end{array}$ \\
\hline $\begin{array}{l}\text { For many particles, the } \\
\text { transmission loss of } \\
\text { information and energy } \\
\text { within the cell disappear }\end{array}$ & $\begin{array}{l}\text { For part of the collective of particles, } \\
\text { viscosity is zero (resistance is zero) }\end{array}$ \\
\hline $\begin{array}{l}\text { A cell can perform its functions } \\
\text { over a certain temperature } \\
\text { range (as well as other } \\
\text { parameters) }\end{array}$ & $\begin{array}{l}\text { Superfluidity (superconductivity) } \\
\text { exists over a certain temperature range } \\
\text { (as well of other parameters, e.g., } \\
\text { magnetic fields) }\end{array}$ \\
\hline $\begin{array}{l}\text { There should be long-range } \\
\text { correlations between } \\
\text { biologically important } \\
\text { molecules }\end{array}$ & $\begin{array}{l}\text { There are long-range correlations } \\
\text { between particles due to the overlap of } \\
\text { their wave functions }\end{array}$ \\
\hline $\begin{array}{l}\text { As a whole, the motion of a } \\
\text { collective of biologically } \\
\text { important molecules in a cell } \\
\text { is quantized? }\end{array}$ & $\begin{array}{l}\text { As a whole, the collective motion of the } \\
\text { particles is quantized }\end{array}$ \\
\hline
\end{tabular}

biologically important molecules in a cell and the phenomena of superfluidity and superconductivity.

It should also be taken into account that so-called "weak measurements" can be implemented under certain conditions that would induce only a partial collapse of the wave function (e.g., Aharonov et al., 1988; Katz et al., 2008). In principle, this fact means that the result of the experiment can be canceled. If such a property can be realized (e.g., at protein folding), we can arrange the experiment such that the wave function collapse would occur only if a native configuration is achieved.

An alternative view of the quantum behavior of biologically important molecules is associated with the many-worlds interpretation of Everett, which is followed by a number of researchers (e.g., Deutsch, 1997). According to this interpretation, the reality in which the protein is properly folded can be selected.

Let us discuss a number of problems in which the quantum behavior of biologically important molecules can be crucial.

The understanding of the stability of intracellular information channels and intracellular computing is critical for the creation of artificial cells. The recognition and transport of molecules inside a cell are the basis of many computational molecular processes. Bray (1995) reviewed these processes and showed that proteins can be considered elements of a computer network. DNA molecules, for example, can be used for DNA computing. However, the problem of the accumulation of errors in the calculation remains unsolved.

The proposed hypothesis may be relevant to the solution of the problem of cellular immunity. This problem requires a separate consideration, but it should be noted that the problem of molecular recognition that was considered in part 1 is crucial for cell-mediated immunity.

We also note that the high transport efficiency of substances applies not only to large molecules but also to ions because transport systems are proteins. The experimental data show that the conversion efficiency of the free energy of ATP to the free energy of ions is approximately 90\% (e.g., Melkikh and Seleznev, 2012). This result implies that it is necessary that energy only be transferred to ions during transport processes to obtain a high efficiency in any intracellular transport process and all other degrees of freedom would be prohibited. This hypothesis is supported by models that describe the process of transport (e.g., Melkikh and Seleznev, 2006a, 2006b, 2012), which demonstrate that high efficiency is only possible if this hypothesis is true. The high efficiency of transport processes can be explained based on the assumption that the proteins in the cell are in a coherent state. The transfer of energy to 
other degrees of freedom may be restricted by quantum selection rules.

The discussed problems in the transmission of information by proteins are compounded if the information storage and retrieval of DNA is considered. DNA is very long, which implies that the process of folding DNA into a three-dimensional structure is more complex than that of proteins. Thus, the proposed model might be useful for the understanding of the processes of DNA folding and unfolding.

Thus, the cell can be represented as a large set (network) of molecular machines that convert energy. In this system, the majority of the degrees of freedom of the biologically important molecules in the cell are frozen and the information channels will function effectively (control signals will be transferred with the lowest cost).

\section{Possible experiments to test the hypothesis}

Let us discuss possible experiments that could be used to test the offered hypothesis of the quantum properties of intracellular channels of information. Several of these experiments have been previously described by other authors.

1. McFadden and Al-Khalili (1999) proposed the use of NMR to register the quantum effects of mutations.

2. Matsuno and Paton (2000) and Davies (2004) offered to use X-ray diffraction to detect the speed of molecular machines. In particular, Davies (2004) offered the following experiments: "Alter the nucleotide mix and determine the effect on the motor speed. Deuterate the nucleotides and measure the motor speed. Observe the motor in real time using a synchrotron source". Synchrotron radiation can be created with a high degree of coherence. Thus, it allows the receipt of a precise picture of the macromolecules of interest through X-ray diffraction. It is also possible to note the scattering of thermal neutrons and to perform experiments on free electrons through the use of X-ray lasers (XFEL, under construction). In the latter case, it is possible to achieve a sufficiently high resolution, both in space and in time, that will allow the "on-line" observation of chemical reactions.

3. Matsuno (2006, 2012) performed experiments on observation of the citric acid cycle in the absence of biological enzymes. " $A$ main motivation for this kind of experimental endeavor is due to the observation that the protective means of a likely reaction cycle could be the reaction cycle itself within the scheme of the relative-state formulation of quantum mechanics since the scheme may provide itself with the built-in software guaranteeing the robustness of a reaction cycle if ever likely even in the absence of the relevant enzymes" (Matsuno, 2012). Matsuno's experiments are important to test assumptions on a quantum nature of the transformation of energy in the cell. It is important to provide experiments on various types of energy transformation in artificial cells or in the absence of biological enzymes.

4. The preparation of proteins with a certain isotope structure could reveal the spin quantum effects in the work of cellular molecular machines.

5. Baldwin et al. (2008) discussed two chains of DNA with identical nucleotide structure that experience an attraction to each other. However, the physical mechanism of attraction remains unclear. It is quite possible that such interaction can be explained by the quantum entanglement of nucleotides. One possible experiment to test this hypothesis is the analysis of the interaction of other biologically important molecules: RNA and proteins. The detection of a dependence of an attractive (or repulsive) force on the structure of molecules (i.e., from the information that is contained in the structures) would be an important proof of the quantum character of the interaction.

6. A number of publications (e.g., Misteli, 2007) describe the localization of chromosomes in a nucleus using methods based on luminescence. In particular, it has been experimentally shown that the arrangement of chromosomes in a nucleus is ordered. In addition, the presence of the distant order of chromosomes has been shown. Such effect could be the consequence of distant quantum correlations of DNA. To confirm this hypothesis, an "on-line" analysis of the positions of the chromosomes is necessary.

7. If quantum correlations exist between proteins in a cell, then the fluctuations of these molecules should be dependent on each other. In this sense, the study of the fluctuations of biochemical reactions, as well as the works of transport systems and other processes, can provide information on such a connection.

8. As has been mentioned above, some proteins are folded incorrectly. The statistics of these incorrectly folded proteins can provide information on how their folding mechanisms are dependent on external conditions (e.g., temperature, $\mathrm{pH}$ and other parameters).

\section{Conclusion}

A problem related to the high efficiency of information and energy transmission in intracellular processes was formulated. Although the number of possible degrees of freedom of biologically important molecules is exponentially large, the mechanisms by which molecular processes maintain high selectivity remains unclear. Therefore, it was proposed that biologically important molecules are found in stable quantum coherent states. An analogy between the quantum properties of biologically important molecules and the phenomena of superfluidity and superconductivity in condensed matter was considered. In addition, a number of experiments that can be used to test the hypothesis were proposed. The aim of these experiments should be the detection of the quantum behavior of biological molecules.

\section{References}

Aharonov, Y., Albert, D.Z., Vaidman, L., 1988. How the result of a measurement of a component of the spin of a spin-1/2 particle can turn out to be 100 . Phys. Rev. Lett. 60, 1351-1354.

Bai, Y., 2003. Hidden intermediates and Levinthal paradox in the folding of small proteins. Biochem. Biophys. Res. Commun. 305 (4), 785-788.

Bai, Y., 2006. Energy barriers, cooperativity, and hidden intermediates in the folding of small proteins. Biochem. Biophys. Res. Commun. 340 (3), 976-983.

Baldwin, G.S., Brooks, N.J., Robson, R.E., Wynveen, A., Goldar, A., Leikin, S., Seddon, J.M., Kornyshev, A.A., 2008. DNA double helices recognize mutual sequence homology in a protein free environment. J. Phys. Chem. B 112 (4), 1060-1064.

Beck, F., Eccles, J.C., 1992. Quantum aspects of brain activity and the role of consciousness. PNAS 89, 11357-11361.

Berezovsky, I.N., Trifonov, E.N., 2002. Loop fold structure of proteins: resolution of Levinthal's paradox. J. Biomol. Struct. Dyn. 20 (1), 5-6.

Bindi, L., Steinhardt, P.J., Yao, N., Lu, P.J., 2009. Natural quasicrystals. Science 324, 1306-1309.

Bray, D., 1995. Protein molecules as computational elements in living cells. Nature 376, 307-312.

Cherstvy, A.G., 2011. Electrostatic interactions in biological DNA-related systems. Phys. Chem. Chem. Phys. 13, 9942-9968.

Davies, P., 2004. Does quantum mechanics play a non-trivial role in life? Biosystems 78, 69-79.

Davies, P., Demetrius, L.A., Tuszynski, J.A., 2012. Implications of quantum metabolism and natural selection for the origin of cancer cells and tumor progression. AIP Adv. 2, 011101.

Deutsch, D., 1997. The Fabric of Reality. Allen Lane, London.

Filmore, D., 2004. It's a GPCR World. Mod. Drug Discovery 7 (11), 24-28.

Finkelstein, A.V., Ptitsyn, O.B., 2002. Protein Physics. Academic Press, Oxford.

Garcia-Viloca, M., Gao, J., Karplus, M., Truhlar, D.G., 2004. How enzymes work: analysis by modern rate theory and computer simulations. Science 303, 186-195.

Gorlich, D., Artmann, S., Dittrich, P., 2011. Cells as semantic systems. Biochem. Biophys. Acta $1810,914-923$. 
Goswami, A., Todd, D., 1997. Is there conscious choice in directed mutation, phenocopies, and related phenomena? An answer based on quantum measurement theory. Physiol. Behav. Sci. 32, 132-142.

Grosberg, A.Y., Khokhlov, A.R., 2010. Giant Molecules: Here, There, and Everywhere, 2nd ed. World Scientific Publishing Company, London.

Hameroff, S., 2003. Conduction pathways in microtubules, biological quantum computation and consciousness. Biosystems 64, 149-168.

Igamberdiev, A.U., 1999. Foundations of metabolic organization: coherence as a basis of computational properties in metabolic networks. Biosystems 50,1-16.

Igamberdiev, A.U., 2004. Quantum computation, non-demolition measurements, and reflective control in living systems. Biosystems 77, 47-56.

Katz, N., Neeley, M., Ansmann, N., Bialczak, R.C., Hofheinz, M., Lucero, E., O'Connell, A., Wang, H., Cleland, A.N., Martinis, J.M., Korotkov, A.N., 2008. Reversal of the weak measurements of a quantum state in a superconductive phase qubit. Phys. Rev. Lett. 101, 200401

King, N., Hittinger, C.T., Carroll, S.B., 2003. Evolution of key cell signaling and adhesion protein families predates animal origins. Science 301 (5631), 361-363.

Macia, E., 2006. The role of aperiodic order in science and technology. Rep. Prog. Phys. 69, 397-441.

Matsuno, K., Paton, R.C., 2000. Is there a biology of quantum information? Biosystems $55,39-46$

Matsuno, K., 2006. Forming and maintaining a heat engine for quantum biology. Biosystems 85, 23-29.

Matsuno, K., 2012. Chemical evolution as a concrete scheme for naturalizing the relative-state of quantum mechanics. Biosystems 109, 159-168.

McFadden, J., Al-Khalili, J., 1999. A quantum mechanical model of adaptive mutation. Biosystems 50, 203-211.
Melkikh, A.V., Seleznev, V.D., 2006a. Requirements on models and models of active transport of ions in biomembranes. Bull. Math. Biol. 68 (2), 385-399.

Melkikh, A.V., Seleznev, V.D., 2006b. Model of active transport of ions in biomembranes based on ATP-dependent change of height of diffusion barriers to ions. J. Theor. Biol. 242 (3), 617-626

Melkikh, A.V., Seleznev, V.D., 2012. Mechanisms and models of the active transport of ions and the transformation of energy in intracellular compartments. Prog. Biophys. Mol. Biol. 109 (1-2), 33-57.

Melkikh, A.V., Sutormina, M.I., 2013. Developing Synthetic Transport Systems. Springer, Dordrecht.

Meszaros, B., Simon, I., Dosztanyi, Z., 2011. The expanding view of protein-protein interactions: complexes involving intrinsically disordered proteins. Phys. Biol. 8, 035003 (10 pp.).

Misteli, T., 2007. Beyond the sequence: cellular organization of genome function. Cell 128, 787-800.

Ogryzko, V.V., 1997. A quantum-theoretical approach to the phenomenon of directed mutations in bacteria (hypothesis). Biosystems 43, 83-95.

Patel, A., 2001. Why genetic information processing could have a quantum basis. J. Biosci. 26 (2), 145-151.

Savir, Y., Tlusty, T., 2007. Conformational proofreading: the impact of conformationa changes on the specificity of molecular recognition. PLOS ONE 2 (5), e468.

Steinhardt, P.J., 2008. How does your quasicrystal grow? Nature 452 (6), 43-44.

Van Kampen, N.G., 2007. Stochastic Processes in Physics and Chemistry, 3d ed. Elsevier, Netherlands.

Waltermann, C., Klipp, E., 2011. Information theory based approaches to cellular signaling. Biochem. Biophys. Acta 1810, 924-932.

Zwanzig, R., Szabo, A., Bagchi, B., 1992. Levinthal's paradox. PNAS 89, 20-22. 